\title{
Directional Measures of Postural Sway as Predictors of Balance Instability and Accidental Falls
}

\author{
by \\ Janusz W. Błaszczyk1,2, Monika Beck1, Justyna Szczepańska , Dorota Sadowska3, \\ Bogdan Bacik', Grzegorz Juras ${ }^{1}$, Kajetan J. Stomka ${ }^{1}$
}

\begin{abstract}
Despite the obvious advantages and popularity of static posturography, universal standards for posturographic tests have not been developed thus far. Most of the center-of-foot pressure (COP) indices are strongly dependent on an individual experimental design, and are susceptible to distortions, which makes results of their analysis incomparable. In this research, we present a novel approach to the analysis of the COP trajectory based on the directional features of postural sway. Our novel output measures: the sway directional indices (DI) and sway vector (SV) were applied to assess the postural stability in the group of young able-bodied subjects. Towards this aim, the COP trajectories were recorded in 100 students standing still for $60 \mathrm{~s}$, with eyes open (EO) and then, with eyes closed (EC). Each record was subdivided then into 20,30 and 60 s samples. Interclass correlation coefficients were calculated from the samples. The controlled variables (visual conditions) uniquely affected the output measures, but only in case of proper signal pretreatment (low-pass filtering). In filtering below $6 \mathrm{~Hz}$, the DI and SV provided a unique set of descriptors for postural control. Both sway measures were highly independent of the trial length and the sampling frequency, and were unaffected by the sampling noise. Directional indices of COP filtered at $6 \mathrm{~Hz}$ showed high to very high reliability, with ICC range of 0.7-0.9. Results of a single $60 \mathrm{~s}$ trial are sufficient to reach acceptable reliability for both DI and SV. In conclusion, the directional sway measures may be recommended as the primary standard in static posturography.
\end{abstract}

Key words: postural stability, sway trajectory, directional measures.

\section{Introduction}

The use of static posturography in the clinical diagnostics is not new, but so far, no widespread consensus has emerged about the methods, techniques and interpretation of the data (Błaszczyk, 2008; Błaszczyk et al., 2014; Duarte and Freitas, 2010; Reymakers et al., 2005). There are extensive attempts to resolve these inconsistencies by searching for adequate and reliable methods of posturographic signal parameterization that would reveal an unambiguous relationship between sway and postural stability (Maki et al., 1994; Piirtola and Era, 2006).
During quiet stance, postural control can be modelled as a continuous process of the stabilization of a multilink inverted pendulum (Maurer and Peterka, 2005; Winter et al., 1996). In static posturography, this process corresponds with maintaining the body's center of mass $(\mathrm{COM})$ at the reference position (RP) within the predefined stability area of the support base (Błaszczyk et al., 1994; Błaszczyk, 2008; Błaszczyk, 2016). Unfortunately, due to numerous nonlinearities and delays within the neuromuscular control system, the COM performs spontaneous oscillations around the RP, known as postural sway.

\footnotetext{
1 - Department of Human Motor Behavior, Jerzy Kukuczka Academy of Physical Education. Katowice Poland.

2 - Department of Neurophysiology, Nencki Institute of Experimental Biology, Warsaw, Poland.

3 - Department of Physical Education and Health Promotion, Szczecin University, Poland.
} 
Natural aging, many pathologies and even eyes closure significantly increase postural sway which seems to be detrimental for postural stability. Generally, greater sway rises the probability of an uncontrolled crossing of stability limits (Błaszczyk et al., 1994; Błaszczyk, 2016). This can happen when the RP placement or the sway amplitude and direction exceed the permissible limits (Błaszczyk, 2016). The clinical manifestation of balance deficiency is increased susceptibility to falls with their often irreversible or even potentially fatal consequences.

Thus far, static posturography remains the most popular method to assess postural stability decline. In this method, the postural control assessment is based on characteristics of the center-of-foot pressure (COP) oscillations, which represent COM motion at the level of the support surface (Błaszczyk, 2008). Unfortunately, there are several limitations of this method (Błaszczyk et al., 2014, Błaszczyk, 2016; Duarte and Freitas, 2010). For instance, it is assumed that recorded COP trajectory is free from measurement distortions that may affect their analysis. There is also a growing body of evidence indicating that incorrect hardware setting (sampling frequency and filtering) may substantially distort the COP signal (Błaszczyk et al., 2014; Błaszczyk, 2016). Several factors, such as an individual experimental design and even the hardware characteristics, should also be considered as potential sources of discrepancy. In particular, the trial length and the number of trials should be considered as factors that may influence the results (Corriveau et al., 2000; Duarte and Freitas, 2010; Pinsault and Vuillerme, 2009). Moreover, most of the commonly analyzed COP output measures are neither sensitive enough to experimental conditions, nor do they exhibit the specific effects for postural deficits (Błaszczyk, 2016; Raymakers et al., 2005). Thus, the standard spatiotemporal analysis of the COP may provide only descriptive information without any direct insight into underlying control deficits.

Recognizing these problems, researchers were compelled to implement more advanced analytical methods in postural sway analysis (Błaszczyk and Klonowski, 2001; Bottaro et al., 2008; Collins and De Luca, 1993; Rocchi et al., 2004; Sabatini, 2000; Kilby and Newell, 2014). The current study is in line with this research and aimed to optimize the application of the COP directional measures in balance assessment (Błaszczyk et al., 2014; Błaszczyk, 2016). Hence, the COP directional characteristics and their sensitivity to visual conditions were tested in young healthy subjects with well-documented efficiency in balance control. The verification of these objectives represents a preliminary step in establishing the feasibility of using static posturography in the assessment of postural stability.

\section{Material and Methods}

The participants in the study consisted of two gender groups of able-bodied subjects: 50 female and 50 male. Basic anthropometric characteristics of the groups are shown in Table 1. All subjects met the ethical requirements for the study and gave their informed consent. They reported having no known neurological or movement disorders and that they engaged in regular physical activity. No further restrictions or participation criteria were used. The research was accepted by the Institutional Bioethics Committee.

During the tests, the subjects were required to maintain a quiet, comfortable posture while standing barefoot on the force platform, with heels aligned at a reference line and arms kept comfortably at the side. Their COP trajectories were recorded by a force plate (Kistler, type 9281C). The data were obtained in six $60 \mathrm{~s}$ trials: 3 with eyes open (EO) and 3 with eyes closed (EC). The COP signals were sampled at 40 $\mathrm{Hz}$ and to remove the noise of signal digitalization they were filtered off-line with the Chebyshev II 10th order low-pass filters (Matlab v. 6.0, The MathWorks, Inc, USA). To assess the optimal filtering, the cut-off frequencies within the range 5-12 Hz were applied.

\section{Computation of the COM and COP directional measures}

In force-platform posturography, the sway trajectory is commonly stored as a rectangular position matrix consisting of $\mathrm{N}$ row position vectors, where $\mathrm{N}$ is the number of data samples collected during a trial. Each COP position is represented by a pair of numerical Cartesian coordinates, $\mathrm{x}$ and $\mathrm{y}$, which corresponds to anatomical directions: AP and ML. The position matrix is then converted to a displacement matrix 
which consists of N-1 displacement values. Each COP displacement specifies changes in the position of a given data point relative to its previous position:

$$
S_{A P}=\left|y_{k}-y_{k-1}\right| \text { and } S_{M L}=\left|x_{k}-x_{k-1}\right|
$$

The sway directional indices (DI) were defined as the ratio of the anteroposterior $\left(S_{A P}\right)$ or the mediolateral $\left(S_{M L}\right)$ path lengths divided by the total COP length (Sтот):

$$
\begin{aligned}
D I_{A P} & =\frac{S_{A P}}{S_{T O T}} \\
D I_{M L} & =\frac{S_{M L}}{S_{T O T}}
\end{aligned}
$$

The data filtered at $6 \mathrm{~Hz}$ were also used to compute the sway vector (SV) coordinates. For this purpose, the displacement coordinates of postural sway were converted to polar coordinates $(\bar{V}, \bar{\varphi})$ according to formulae (4) and (5). The average COP velocity i.e. the length (or the magnitude) of the $\mathrm{SV}$ could be easily calculated by taking the total COP path length ( $\left.S_{c o p}\right)$ during a trial and dividing it by the time of the trial $\left(T_{\text {trial }}\right)$ :

$$
\bar{V}_{c o p}=\frac{S_{\text {cop }}}{T_{\text {rrial }}}=\frac{F_{s}}{N} \sum_{k=2}^{N} \sqrt{\left(x_{k}-x_{(k-1)}\right)^{2}+\left(y_{k}-y_{(k-1))^{2}}\right.}
$$

where: $\mathrm{N}$ is the total number of data points for a given trial length (Ttrial) and $F_{s}$ is the sampling frequency of an A-to-D converter

The azimuth (or the COP polar angle) of the SV was computed according to the following formula:

$$
\varphi=\arctan \frac{S_{A P}}{S_{M L}}=\arctan \frac{V_{A P}}{V_{M L}}=\arctan \frac{D I_{A P}}{D I_{M L}}
$$

The low-pass filtering at $0.4 \mathrm{~Hz}$ allowed us the assessment of the COM trajectory from the COP data (Błaszczyk, 2008). All the above computational formulae hold true for calculating the COM DIs and SV coordinates.

\section{Statistical analysis}

All statistical analyses in this study were performed using the Statistica version 6.0 software (StatSoft, Inc. USA). Repeated measures analysis of variance (group $\mathrm{x}$ vision $\mathrm{x}$ filter $2 \times 2 \times 7$ ANOVA with two dependent factors: vision and filtering frequency) followed by a post-hoc LSD test (Fisher's Least Significant Difference test) was used to examine differences in the analyzed directional indices. Significance was accepted at $p$ $<0.05$. Next, interclass correlation coefficients
(ICC) were computed to assess reliability of the directional measures.

\section{Results}

One-way analysis of variance showed highly significant differences between the male and female groups in body mass and height as well in the size of their base of support. Descriptive statistics of the analyzed anthropometric variables are summarized in Table 1.

In the following analysis, the effects of gender (male vs. female), direction (AP vs. ML) and visual conditions (EO vs. EC) on postural sway indices were tested. The subject's gender had no effect on the DIML. ANOVA showed significant effects of two categories: vision $\left(\mathrm{F}_{1,98}=\right.$ $32.7, p \leq 0.0001)$ and the filter cut-off frequency $\left(\mathrm{F}_{6,588}=119, p \leq 0.00001\right)$ on the mediolateral directional index. The DIML values depended significantly on the filtering frequency and the lowest value of this variable was noticed for filtering frequency of $11 \mathrm{~Hz}(0.51 \pm 0.03$ for eyes open tests and $0.50 \pm 0.04$ in EC trials). For filtering at $5 \mathrm{~Hz}$, the mediolateral directional index increased up to $0.56 \pm 0.06$ in EO trials and to $0.53 \pm 0.07$ in EC tests. However, the group $\mathrm{x}$ vision $x$ filter interactions reached the level of significance $\left(\mathrm{F}_{6,588}=4.36, p \leq 0.001\right)$ documenting different dynamics of the increase within each tested group and the visual conditions. In particular, young male subjects while tested without vision demonstrated significantly lower values of the DIML. Details of these analyses are shown in Figure 1.

The subject's gender had no effect on the antero-posterior directional index $(p \leq 0.34)$, but ANOVA indicated significant effects of two categories: vision $\left(\mathrm{F}_{1,98}=31.5, p \leq 0.0001\right)$ and the filter cut-off frequency $\left(\mathrm{F}_{6,588}=141.6, p \leq 0.00001\right)$ on the DIAP index. The DIAP values decreased significantly with the cut-off frequency of the Cheby filters and the highest value of this variable was found for filtering frequency of $11 \mathrm{~Hz}(0.75 \pm$ 0.03 for EO testing and slightly increased in EC trials to $0.76 \pm 0.03$ ). For filtering at $5 \mathrm{~Hz}$, the DIAP values dropped to $0.70 \pm 0.05$ and to $0.73 \pm 0.06$ in EO and EC trials, respectively. Similarly, as in the lateral DI, the group $\mathrm{x}$ vision $\mathrm{x}$ filter interactions in the DIAP also crossed the level of significance $\left(\mathrm{F}_{6,588}=3.18, p \leq 0.01\right)$ indicating a different 
changes of the DIAP due to filtering within each tested group and depending on the visual conditions. As in case of the lateral DI, young male subjects while standing with eyes closed showed significantly different values of the DIAP compared to the female subjects. Their values stayed at the level of $0.74 \pm 0.06$ when COP data were filtered at $5 \mathrm{~Hz}$ and increased to $0.76 \pm 0.03$. Details of this analysis are depicted in Figure 2.

Table 1

Group characteristics (mean $\pm S D$ ) of female and male subjects.

$L$ - length and W- width of the BOS (base of support). NS - nonsignificant

\begin{tabular}{lccccc}
\hline Group $($ size $)$ & Age $(\mathrm{yrs})$ & Height $(\mathrm{cm})$ & Body mass $(\mathrm{kg})$ & L-BOS $(\mathrm{cm})$ & W-BOS $(\mathrm{mm})$ \\
\hline Male $(\mathrm{n}=50)$ & $21.2 \pm 2.0$ & $180.1 \pm 7.2$ & $77.2 \pm 8.7$ & $27.2 \pm 1.4$ & $26.4 \pm 1.3$ \\
Female $(\mathrm{n}=50)$ & $20.7 \pm 1.8$ & $167.3 \pm 6.2$ & $58.8 \pm 7.1$ & $24.8 \pm 1.1$ & $24.2 \pm 2.2$ \\
$F_{1,98,} p<0.0001$ & NS & 92.3 & 134.0 & 90.1 & 37.4 \\
\hline
\end{tabular}

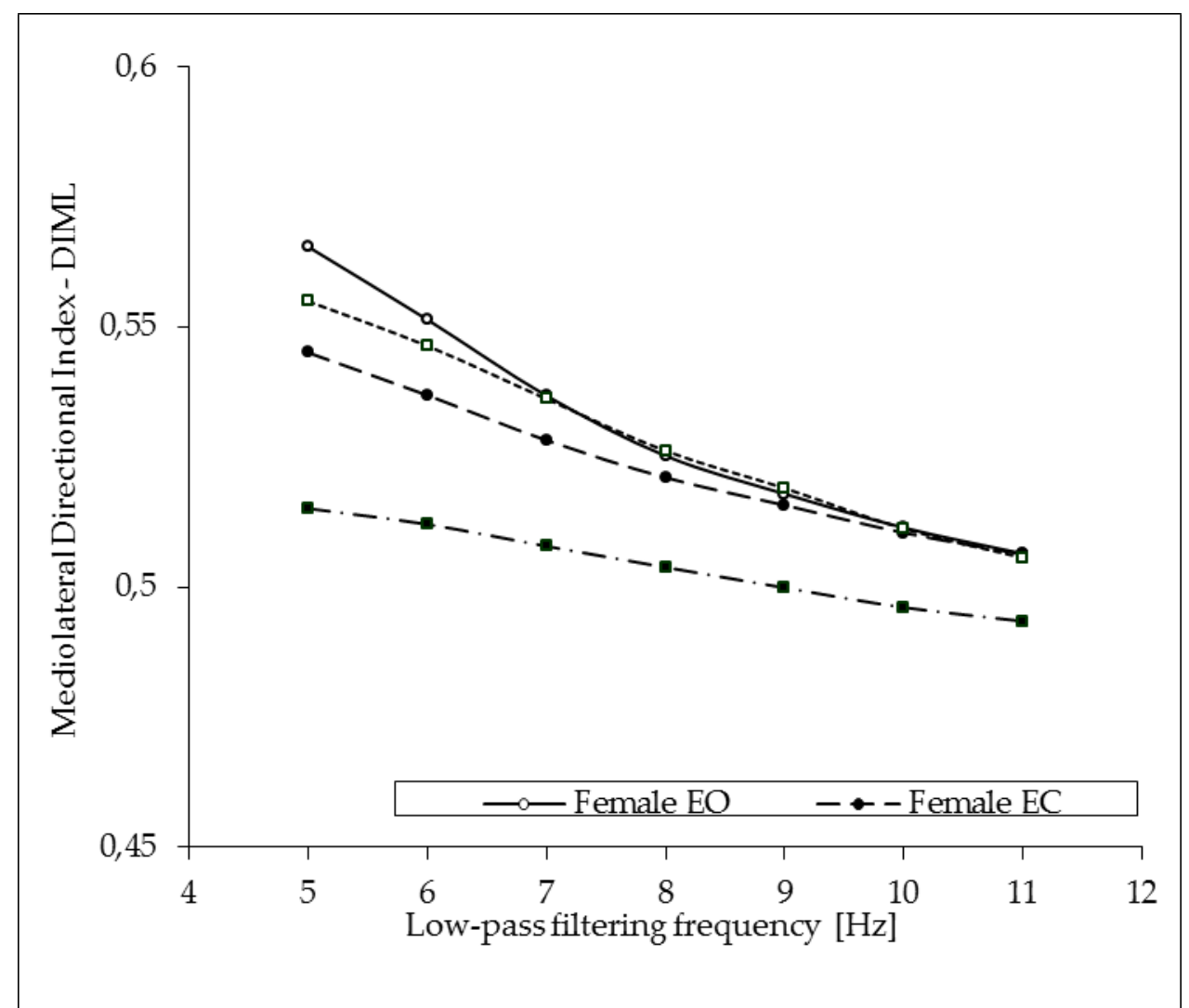

Figure 1

Impact of the COP signal low-pass filtering on the medio-lateral directional sway indices (DIML) in young male $(M)$ and female $(F)$ subjects standing quiet with eyes open (EO) and with eyes closed (EC). 


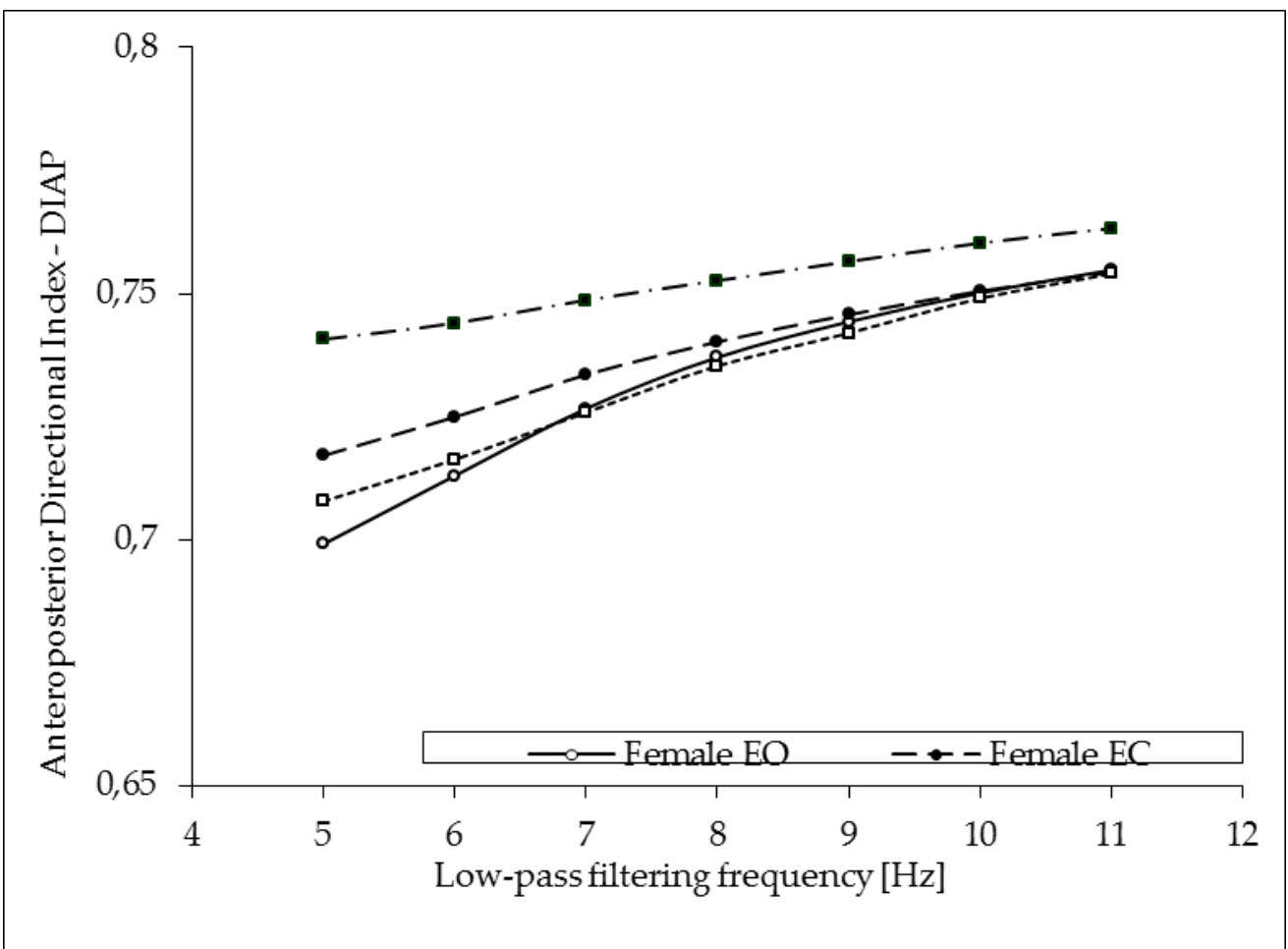

Figure 2

Impact of the COP signal low-pass filtering on the antero-posterior directional sway indices (DIAP) in young male (M) and female $(F)$ subjects standing quiet with eyes open (EO) and with eyes closed (EC).

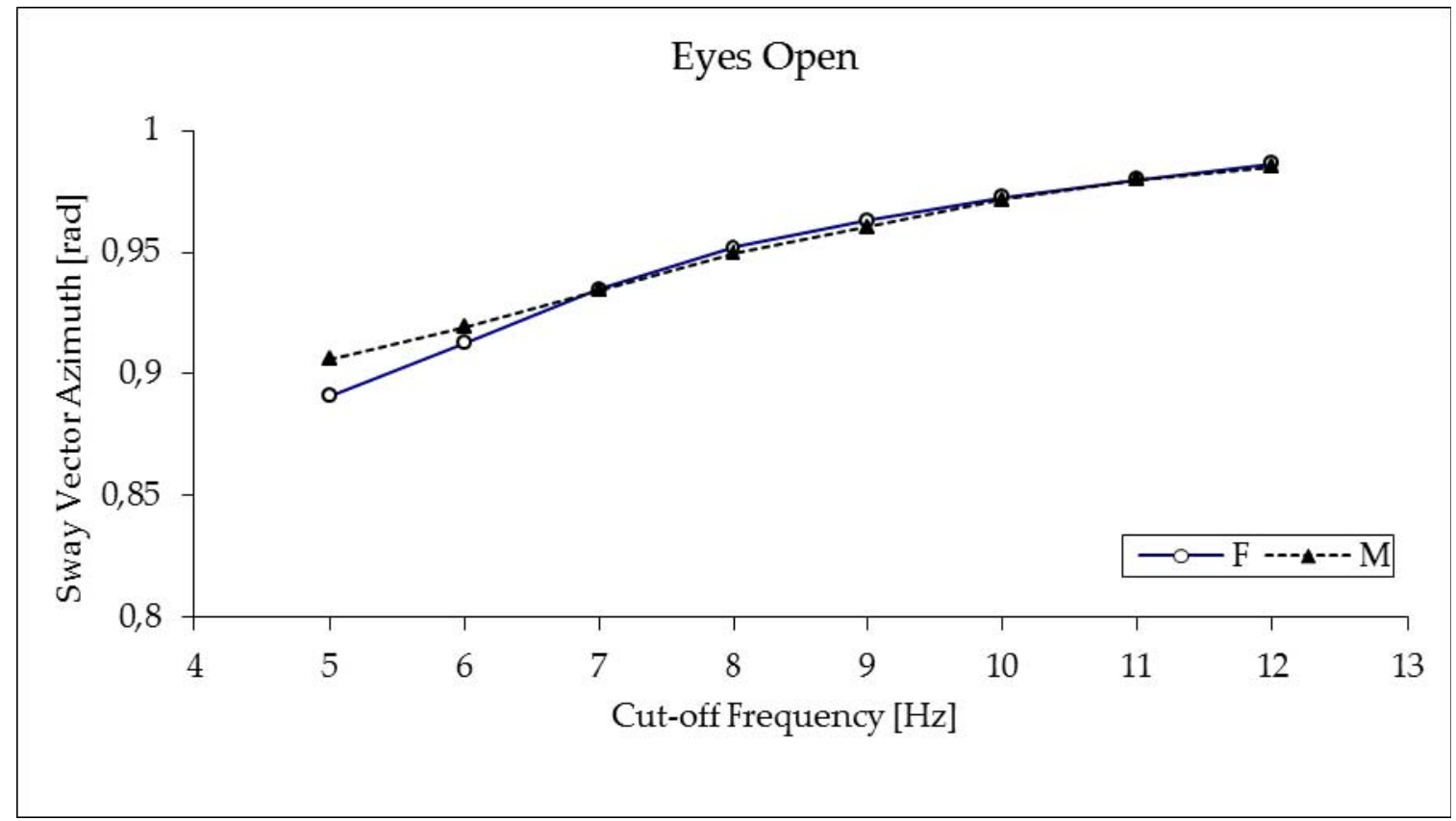

Figure 3

Impact of the COP signal low-pass filtering on the sway vector azimuth in young male $(M)$ and female $(F)$ subjects standing quiet with eyes open (EO). The error bars represent standard deviations. 


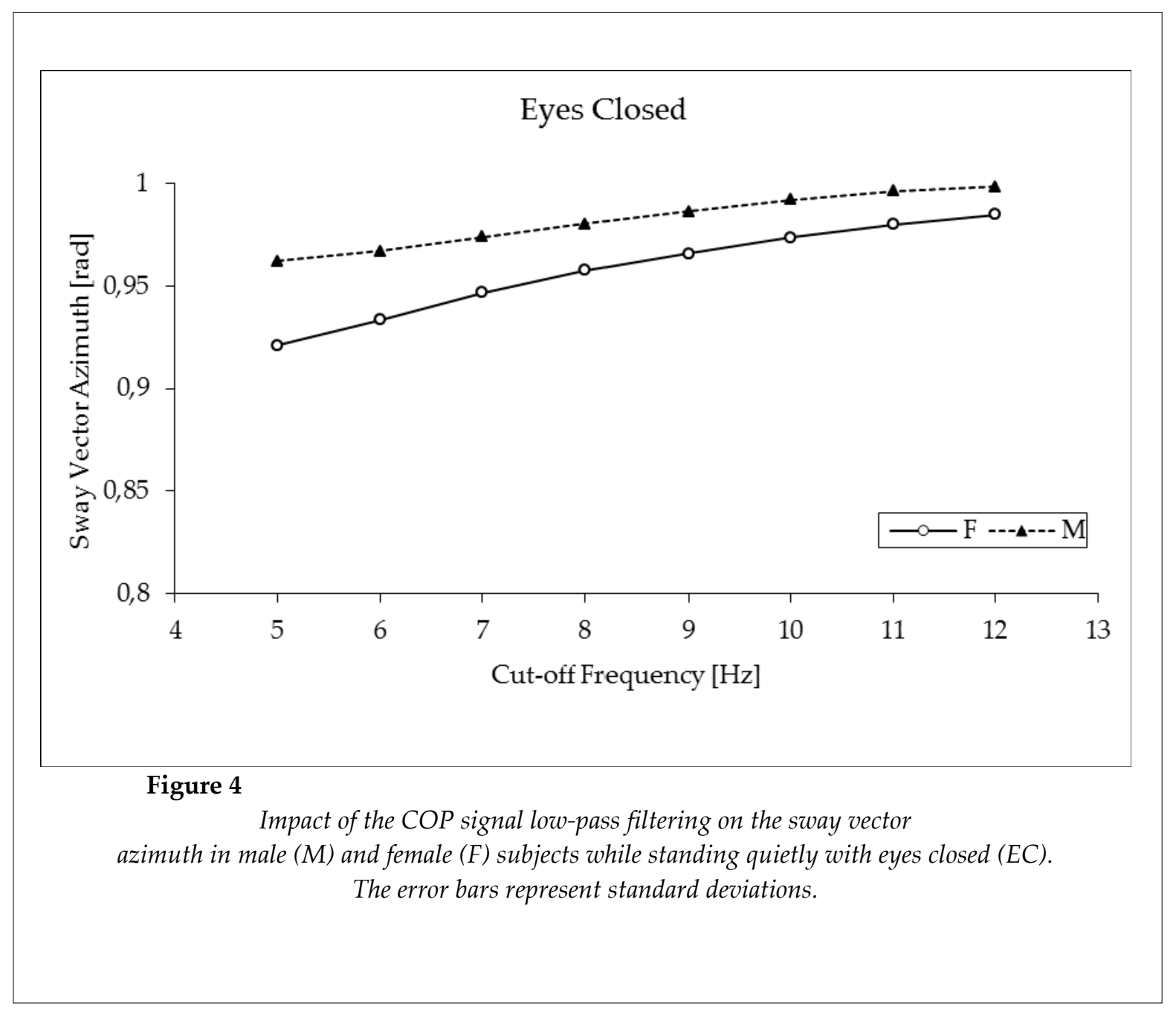

Table 2

Validation results for Directional Indices: antero-posterior (DIAP), mediolateral (DIML). Interclass correlation (ICC) analysis for two-way mixed effects model. $E O$-eyes open tests, EC-eyes closed tests, in the female $(F)$ and male $(M)$ group of subjects

\begin{tabular}{|c|c|c|c|c|c|}
\hline & \multicolumn{3}{|c|}{$\operatorname{ICC}(2,1)$} & \multirow{2}{*}{$95 \%$ CI } & \multirow{2}{*}{ ICC 95\% CI } \\
\hline & 0.7 & 0.8 & 0.9 & & \\
\hline $\mathrm{EO}$ & 1 & 2 & 3 & $0.870-0.961$ & $0.676-0.803$ \\
\hline EC & 1 & 1 & 2 & 0.903-0.997 & $0.716-0.830$ \\
\hline
\end{tabular}




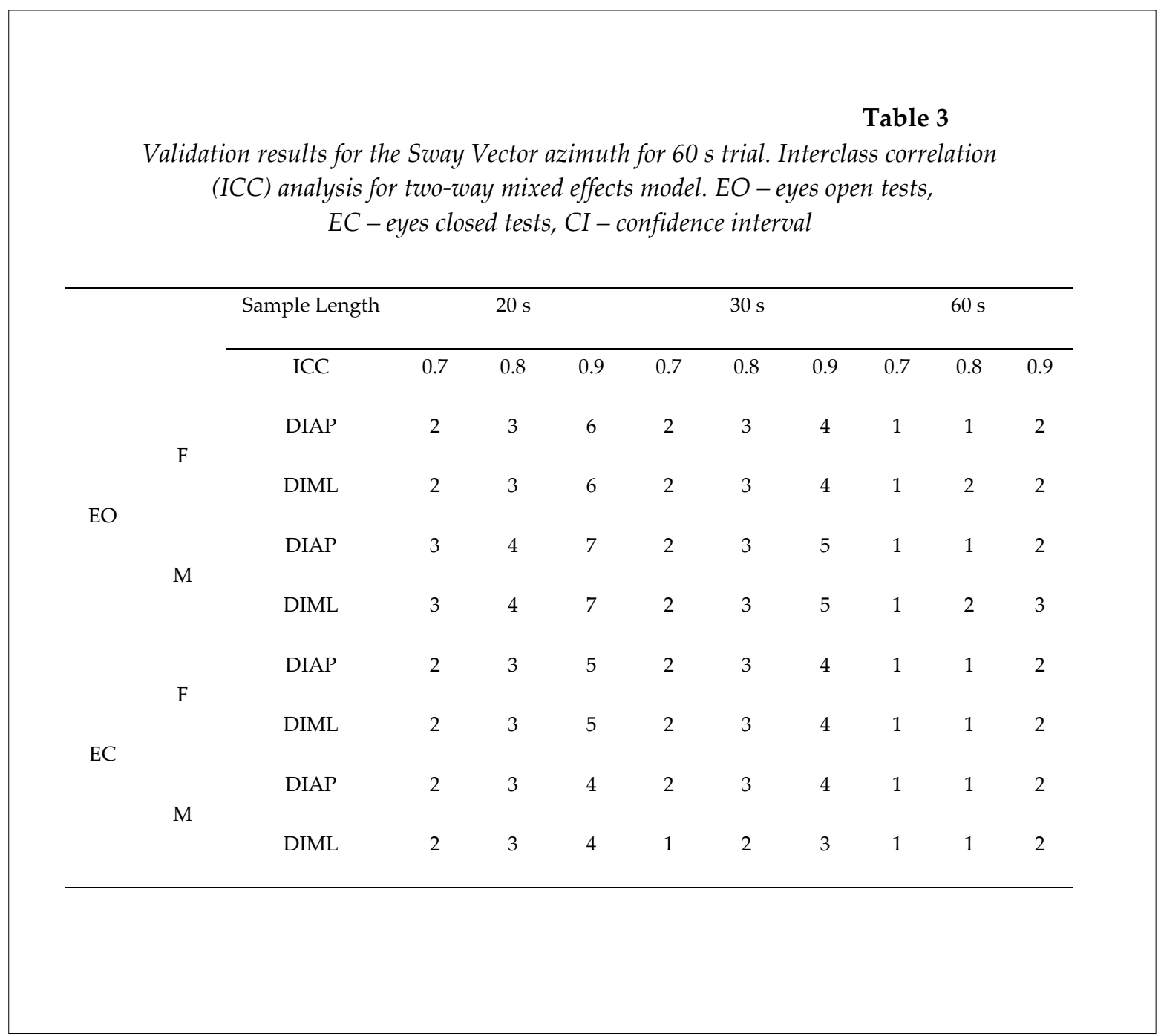

As expected, the sway vector azimuth exhibited some dependency on gender and filtering frequency in EC trials (for details see Figures 3 and 4). The ANOVA, however, showed no difference between the tested groups (male vs. female) and no effects of filtering. Therefore, further analysis was focused on sway vector azimuth values assessed by filtering at $5 \mathrm{~Hz}$. Twoby-two ANOVA with subjects' sex as an independent measure and vision as a repeated measure factor confirmed no effect of group ( $\mathrm{p} \leq$ 0.13 ) and documented both a significant effect of vision $(\mathrm{F} 1,98=82.1, \mathrm{p} \leq 0.00001)$ and significant interaction group $\mathrm{x}$ vision $(\mathrm{F} 1,98=7.97, \mathrm{p} \leq 0.01)$. In the male group, higher dependency on vision was observed, while in the female group, eyes closure resulted only in a slight but significant ( $p$ $\leq 0.0005$ ) increase of the sway vector azimuth from $0.89 \pm 0.1 \mathrm{rad}(\mathrm{EO})$ to $0.92 \pm 0.1 \mathrm{rad}(\mathrm{EC})$; in male young subjects the effect of vision was more pronounced. Standing quiet with eyes open, male subjects showed the SV azimuth at the level of $0.91 \pm 0.1$, that was significantly higher compared to female subjects $(\mathrm{p} \leq 0.05)$ and significantly less compared to male subjects tested with eyes closed $(0.96 \pm 0.01, \mathrm{p} \leq 0.00001)$.

Results of the interclass correlation (ICC) analysis are shown in Tables 2 and 3. The present result showed that a single $60 \mathrm{~s}$ trial was sufficient to measure directional indices and the sway vector azimuth with good reliability level of 0.7 . Shorter trials would require at least two repetitions of a test both with eyes open and with eyes closed.

\section{Discussion}

This study aimed to quantify the characteristics of spontaneous postural sway in 
order to establish reliability of our novel directional measures that can be used in assessment of postural instability. For this purpose, the COP trajectories were recorded during a quiet stance (with eyes open and then with eyes closed) in young healthy subjects, known from the most efficient balance control. Their COP characteristics were analyzed using several recently introduced measures: sway directional indices (Błaszczyk et al., 2014) and the sway vector (Błaszczyk, 2016). These measures showed several advantages, making them unique in the assessment of postural control (Błaszczyk, 2016). They were virtually independent of such important factors as the number of trials and their length, as well as the sampling frequency of COP trajectory. The present study showed that reliability of the postural stability assessment was critically dependent on COP data pretreatment. In this context, the most comprehensive measure proved to be both DI and SV. In the latter case, the SV attributes, however, were affected by the initial COP data filtering.

The SV concept is an extension of the stability radius applied, which in basic terms describes the largest magnitude of perturbation that might be tolerated by postural control without loss of balance. The probability of successful balance recovery in the face of perturbation depends on three attributes that define the sway vector. An excessive magnitude of the sway vector increases uncertainty in postural control, substantially reduces the time for balance recovery action, and thus, increases the probability of a fall (Błaszczyk, 2016). Therefore, it is crucial for postural stability analysis to remove by proper filtering all distortions resulting from the sway data collection. Present results suggest that in young healthy subjects low-pass filtering at $6 \mathrm{~Hz}$ is the most effective. It removes noise without affecting the sway trajectories.

The timing of the recovery action depends also on the direction of the perturbation (Błaszczyk et al., 1994, 2014; Błaszczyk, 2016), thus, the SV azimuth seems to be another valuable attribute of the sway vector. This attribute is a derivative of the DI and similarly to those measures it allows to analyze postural control mechanisms. It is well established that two separate mechanisms must be considered for postural control in the AP and ML directions (Winter et al., 1996), therefore, each axis would correspond to a different stability radius (Błaszczyk et al., 2014; Błaszczyk, 2016). These mechanisms are, however, mutually interrelated by the object of the control. They are both acting on the same inverted pendulum of the human body. This interaction is shown here by the SV azimuth.

Directionality and asymmetry of sway characteristics imply the asymmetry of both the postural control and the stability area. Our present results of DI and the SV azimuth in young healthy subjects document that, to maintain the most stable posture, the neuromuscular system must allocate more effort to control AP stability. This can be seen in the mean value of both directional indices (DIAP $=0.77$ vs DIML $=0.49$ ). The invariant value of the SV azimuth $(0.93$ radian) in these subjects also documents this feature of postural control. It is significant, however, that measures in young subjects are rigorously controlled regardless of the visual input. This fixed angle and the SV set the optimal level of interaction between the AP and ML controls, which may be considered as valid determinants of stable posture.

In conclusion, our directional measures (SV and DI) proved their salient features as well as their specificity and sensitivity to visual conditions. It appears that these sway measures can provide a thorough assessment of postural control, particularly in terms of biomechanical and physiological characteristics; and that the output of the posturographic measures can be readily interpreted. When tested, the measures provided a reliable standard in the assessment of postural stability. These results demonstrate proof of the SV concept and allow us to recommend the sway vector as a standard measure in both the laboratory and clinical assessment of postural control.

\section{Acknowledgements}

This research was supported by the statutory funds from the Jerzy Kukuczka Academy of Physical Education in Katowice. 


\section{References}

Błaszczyk J.W, Lowe DL, Hansen PD. Ranges of postural stability and their changes in the elderly. Gait Posture, 1994; 2: 11-17

Błaszczyk JW, Klonowski W. Postural stability and fractal dynamics. Acta Neurobiol Exp, (Warsaw) 2001; 61: $105-112$

Błaszczyk JW. Sway ratio - a new measure for quantifying postural stability. Acta Neurobiol Exp, (Warsaw) 2008; 68: 51-57

Błaszczyk JW, Beck M, Sadowska D. Assessment of postural stability in young healthy subjects based on directional features of posturographic data: vision and gender effects. Acta Neurobiol Exp, (Warsaw) 2014; 74: $433-442$

Błaszczyk JW. The use of sway vector for the assessment of postural instability. Gait Posture, 2016; 44: 1-6, doi: 10.1016/j.gaitpost.2015.10.014

Bottaro A, Yasutake Y, Nomura T, Casadio M, Morasso P. Bounded stability of the quiet standing posture: An intermittent control model. Hum Mov Sci, 2008; 27: 473-495

Collins JJ, De Luca CJ. Open-loop and closed-loop control of posture: A random walk analysis of center of pressure trajectories. Exp Brain Res, 1993; 95: 308-18

Corriveau H, Hebert R, Prince F, Raiche M. Intrasession reliability of the "center of pressure minus center of mass" variable of postural control in healthy elderly. Arch Phys Med Reh, 2000; 81: 45-48

Duarte M, Freitas SMSF. Revision of posturography based on force plate for balance evaluation. Rev Bras Fisioter, 2010; 14: 183-92

Kilby MC, Newell KM. Postural instability detection: aging and the complexity of spatial-temporal distributional patterns for virtually contacting the stability boundary in human stance. Plos One, 2014; 9, doi:10.1371/journal.pone.0108905

Maki BE, Holliday PJ, Topper AK. A prospective study of postural balance and risk of falling in an ambulatory and independent elderly population. J Gerontol Med Sci, 1994; 49: M72M84

Maurer C, Peterka RJ. A new interpretation of spontaneous sway measures based on simple model of human postural control. J Neurophysiol, 2005; 93: 189-200

Piirtola M, Era P. Force Platform Measurements as Predictors of Falls among Older People - A Review. Gerontology, 2006; 52: 1-16

Pinsault N, Vuillerme N. Test-retest reliability of centre of foot pressure measures to assess postural control during unperturbed stance. Med Eng Phys, 2009; 31: 276-286

Raymakers JA, Samson MM, Verhaar HJJ. The assessment of body sway and the choice of the stability parameter(s). Gait Posture, 2005; 21: 48-58

Rocchi L, Chiari L, Cappello A. Feature selection of stabilometric parameters based on principal component analysis. Med Biol Eng Comput, 2004; 42: 71-79

Sabatini AM. Analysis of postural sway using entropy measures of signal complexity. Med Biol Eng Comput, 2000; 38: 617-624

Saripalle SK, Paiva GC, Cliett TC, Derakhshani RR, King GW, Lovelace CT. Classification of body movements based on posturographic data. Hum Mov Sci, 2014; 33: 38-50

Winter DA, Prince F, Frank JS, Powell C, Zabjek KF. Unified theory of A/P and M/L balance in quiet stance. J Neurophysiol, 1996; 75: 2334-2343

\section{Corresponding author:}

\section{Kajetan J. Słomka}

Department of Human Motor Behavior, The Jerzy Kukuczka Academy of Physical Education

72A Mikolowska Str., 40-065 Katowice, Poland

Phone: +48 322075141

Fax: +48322075200

E-mail: k.slomka@awf.katowice.pl 